\title{
COST-EFFECTIVENESS ANALYSIS OF CARDIAC RESYNCHRONIZATION THERAPY IN PATIENTS WITH NYHA I AND NYHA II HEART FAILURE IN SPAIN
}

Luis Almenar

Cardiology Department, Hospital Universitario La Fe

Beatriz Díaz

Cardiology Department, Hospital Universitario Central de Asturias

Aurelio Quesada

Cardiology Department, Hospital General de Valencia

Carlos Crespo

Department of statistics, University of Barcelona Oblikue Consulting
Health Economics and Reimbursement, Medtronic Ibérica

Stuart Mealing

Oxford Outcomes

Cecilia Linde Department of Cardiology, Karolinska University Hospital

Claude Daubert

Département de Cardiologie et maladies vasculaires, CHU

Objectives: The aim of the study was to combine clinical results from the European Cohort of the REVERSE study and costs associated with the addition of cardiac resynchronization therapy (CRT) to optimal medical therapy (OMT) in patients with mild symptomatic (NYHA I-II) or asymptomatic left ventricular dysfunction and markers of cardiac dyssynchrony in Spain.

Methods: A Markov model was developed with CRT + OMT (CRT-ON) versus OMT only (CRT-OFF) based on a retrospective cost-effectiveness analysis. Raw data was derived from literature and expert opinion, reflecting clinical and economic consequences of patient's management in Spain. Time horizon was 10 years. Both costs (euro 2010) and effects were discounted at 3 percent per annum.

Results: CRT-ON showed higher total costs than CRT-OFF; however, CRT reduced the length of hospitalization in ICU by 94 percent ( 0.006 versus 0.091 days) and general ward in by 34 percent ( 0.705 versus 1.076 days). Surviving CRT-ON patients (88.2 percent versus 77.5 percent) remained in better functional class longer, and they achieved an improvement of 0.9 life years (LYGs) and 0.77 years quality-adjusted life years (QALYs). CRT-ON proved to be cost-effective after 6 years, except for the 7th year due to battery depletion. At 10 years, the results were $€ 18,431$ per LYG and $€ 21,500$ per QALY gained.

Probabilistic sensitivity analysis showed CRT-ON was cost-effective in 75.4 percent of the cases at 10 years.

Conclusions: The use of CRT added to OMT represents an efficient use of resources in patients suffering from heart failure in NYHA functional classes I and II.

Keywords: Cardiac resynchronization therapy, REVERSE, Cost-effectiveness analysis, Left ventricle, Heart failure

According to the European Society of Cardiology (ESC) there are at least 15 million patients with heart failure (HF) in a total population of 800 million people in Europe (1). The prevalence of $\mathrm{HF}$ ranges between 2 and 3 percent, and rises sharply at 75 years of age, being the prevalence in 70 - to 80 -year-old patients between 10 and 20 percent (1). HF is responsible for a 5 percent of acute hospital admissions; it is present in 10 percent of patients in hospital beds, and accounts for approximately 2 percent of national expenditure on health, mostly due to the cost of hospital admissions. Overall, 50 percent of patients with HF die after 4 years; moreover, 40 percent of patients admitted to hospital die or are readmitted within 1 year (1).

In Spain, at least 2 percent of the population over 40 years old suffers from HF, 6 to 10 percent of those patients being over 60 years old (2). The three leading causes of death in Spain in
2008 classified by large groups were cardiovascular disease (responsible for 31.7 percent of all deaths), tumors (26.9 percent), and respiratory system diseases (11.4 percent) (3). Specifically, $\mathrm{HF}$ is the third leading cause of cardiovascular death after heart disease and cerebrovascular disease. Additionally, it is the only cardiovascular disease that currently presents a continuous increase in incidence and prevalence (4). From 2000 to 2007, the prevalence of HF increased steadily from 895 to 2,126 per 100,000 person-years, and the incidence augmented from 296 per 100,000 person-years in 2000 to 390 per 100,000 personyears in 2007 (5). It is a progressive and fatal disorder which has an unsatisfactory prognosis even with proper treatment.

Drug therapy for HF has used multiple strategies that include and combine angiotensin-converting enzyme inhibitors, angiotensin II receptor antagonists (or angiotensin receptor 
blockers), aldosterone antagonists and beta blockers. However, a large number of patients have a poor quality of life and present high mortality rates. They are non-responders despite adequate medication $(6 ; 7)$. New targets for therapy are being identified, many of which are focused on specific groups of HF patients (HF with preserved ejection fraction or with chronic kidney disease or with Nephropathy Due to Type 2 Diabetes) rather than the whole population. Cardiac resynchronization therapy (CRT), which aims to restore synchronous cardiac contraction, is one such therapy that attempts to treat patients with cardiac dyssynchrony $(1 ; 7)$. It has been estimated that 20 to 30 percent of patients with symptomatic HF and 10 percent of patients with no selected HF suffer from intraventricular conduction disorders and could benefit from CRT (8).

CRT has been shown to be clinically effective in patients with New York Heart Association (NYHA) classes III or IV HF, left ventricular dysfunction and prolonged QRS duration (1). In addition, recent trials have confirmed the clinical efficacy of CRT in patients with either asymptomatic or mild HF (NYHA I/II), with evidence that CRT may prevent disease progression by reducing clinical outcomes through reverse left ventricular remodeling $(9 ; 10)$. Specifically, the REVERSE study has demonstrated that CRT improves clinical response in NYHA functional class (I-II), included in this composite response structure and function of the left ventricle in mildly symptomatic or asymptomatic patients with left ventricular dysfunction and markers of cardiac dyssynchrony. CRT has demonstrated to be cost-effective in this patient group from the British perspective, with incremental cost-effectiveness ratios (ICER) ranging from $€ 7,538$ to $€ 18,017$ (11).

In this sense, we aim to assess the efficiency of CRT among this population by conducting an economic analysis of CRT based on the REVERSE study from the Spanish Health System perspective.

\section{METHODS}

A cost-effectiveness analysis of cardiac resynchronization versus conventional (optimal medical therapy, OMT) care has been performed using a Markov model based on data from the European REVERSE study, data from the literature and expert opinion to allocate the economic consequences of each option through a 10 -year comparison.

The REVERSE study was designed to validate whether CRT in combination with OMT is beneficial in mildly symptomatic (NYHA I-II) or asymptomatic patients with left ventricular dysfunction and markers of cardiac dyssynchrony to prevent disease progression in the long term. The study was undertaken in centers of the United States and Europe but only the latter performed a 2-year follow-up.

In this European cohort, a total of 287 participants were randomized (2:1) either to the CRT group (CRT-ON) $(n=180)$ or control group (CRT-OFF) $(n=82)$, and followed-up for 24 months.

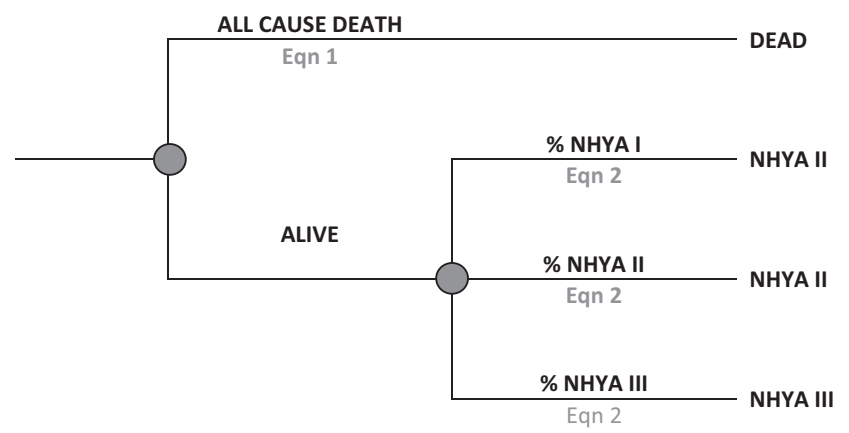

Figure 1. Markov chain used to model the 10-year follow-up period. NYHA, New York Heart Association classification.

Regardless of the assigned group, all patients were treated with OMT in accordance with HF guidelines, and after assessing the appropriateness of participation in the study, defibrillators were implanted to those patients who needed them (ICD / CRT-D).

\section{Study Design}

Type of Analysis. A cost-effectiveness analysis was carried out to compare the alternative scenarios (with/without CRT) using two different effectiveness indicators: life years gained (LYG) and quality-adjusted life-years (QALY) taking into account that different strategies were offered depending on whether patients needed to have defibrillators implanted or not. Data on healthcare resources were adjusted from the REVERSE-EU study with an expert panel consisting of three Spanish cardiologists from different institutions. Clinical opinion was compiled using a structured questionnaire in two interviews, the first exploratory and the second for validation and consensus. Clinical authors were selected according to clinical experience and national and international research achievements. To retain internal consistency, this dataset was analyzed considering a 24month randomized follow-up. Results were finally displayed as cost per LYG and cost per QALY gained with or without CRT (CRT-ON versus CRT-OFF).

The analysis was performed from the Spanish National Health System perspective and only direct medical costs were included over a 10-year time horizon. Both costs and outcomes were discounted at 3 percent per annum.

Modeling. A decision analytic model was developed to estimate the costs and the underlying disease process of patients with mildly asymptomatic HF. Health states were defined by NYHA class (I-III) and death. NYHA class IV was not included in the model because no patient was classified as being in NYHA IV at any follow-up visit. Costs and utilities were attached to each health state and patients in each treatment arm accrued costs and benefits as they passed through these states. Time was measured in discrete units of 1 month (1-month cycles) and a 10 -year time horizon was used (Figure 1). Costs were expressed in Euro 2010. 
Table 1. Unit Cost by Resource Used (Base Case and Range for Sensitivity Analysis)

\begin{tabular}{|c|c|c|c|c|}
\hline & CRT & CRT-D & $I C D$ & Source \\
\hline Device Costs & $\begin{array}{l}€ 4,544.00 \\
(€ 4,433.66-€ 5,792.81)\end{array}$ & $\begin{array}{l}€ 20,259.00 \\
(€ 20,017.79-€ 21,479.08)\end{array}$ & $\begin{array}{l}€ 17,263.77 \\
(€ 16,513.56-€ 17,745.88)\end{array}$ & (13) \\
\hline Leads & $\begin{array}{l}€ 1,605.00 \\
(€ 1,444.50-€ 1,765.50)\end{array}$ & $\begin{array}{l}€ 2,683.00 \\
(€ 2,414.70-€ 2,951.30)\end{array}$ & - & Internal data Medtronic \\
\hline Initial implantation & $\begin{array}{l}€ 2,729.07 \\
(€ 2,577.01-€ 3,936.16)\end{array}$ & $\begin{array}{l}€ 2,729.07 \\
(€ 2,577.01-€ 3,936.16)\end{array}$ & $\begin{array}{l}€ 711.66 \\
(€ 640.50-€ 782.83)\end{array}$ & $\begin{array}{l}\text { (14) Base case CRT/CRT -D is an average } \\
\text { of DRG } 116,115 \text { and } 851 \text {. Base Case } \\
\text { ICD is DRG } 849\end{array}$ \\
\hline Device replacement procedure & $\begin{array}{l}€ 963.52 \\
(€ 770.81-€ 1,156.22)\end{array}$ & $\begin{array}{l}€ 963.52 \\
(€ 770.81-€ 1,156.22)\end{array}$ & $\begin{array}{l}€ 711.66 \\
(€ 640.50-€ 782.83)\end{array}$ & $\begin{array}{l}\text { (14). Base case CRT/CRT-D is an average } \\
\text { of DRG } 118,115 \text { and } 851 \text {. Base Case } \\
\text { ICD is DRG } 849\end{array}$ \\
\hline ICU ward (per day) & & $€ 1,470.10(€ 1,127.25-€ 1,764.12)$ & & $(14)$ \\
\hline General ward (per day) & & $€ 723.56$ (€578.85-€868.27) & & (14) \\
\hline Cardiology visits (per visit) & & $€ 59.69(€ 47.75-€ 71.63)$ & & (14) \\
\hline GP visits (per visit) & & $€ 23.95$ (€20.10-€27.80) & & $(14)$ \\
\hline Hospitalization Costs & & $€ 3,362.29(€ 3,320.61-€ 3,389.19)$ & & (14) DRG 127 \\
\hline
\end{tabular}

CRT, cardiac resynchronization therapy; CRT-D, cardiac resynchronization therapy + defibrillator; ICD, implantable cardioverter defibrillator; ICU, intensive care unit; GP, general practice; DRG: diagnosis-related group.

The whole analytical model was developed in Microsoft Office Excel 2007 (Microsoft Corporation, Redmond, WA) using a three stage process, although regressions were calculated in SAS v9.1 (SAS Institute Inc., Cary, NC) using data from the REVERSE study. First, at any given time point, the surviving proportion of the original cohort was derived. Second, for the same time point the distribution across three NYHA classes was derived for all surviving patients. Finally, the derived proportions in each health state were multiplied by the relevant cost and utility estimates to derive monthly values. All totals were summed over the time horizon to generate total intervention values.

\section{Model Parameters}

Probabilities. A multinomial logistic regression was used to estimate NYHA mix over time. NYHA class was the dependent variable in all analyses and, in addition to a constant term, analyses were carried out using different predictor variables - time, time squared, exp(time), $\log$ (time). All analyses were clustered by patient. On the basis of a visual check of goodness-of-fit to the within-trial data, and the opinion of the project steering committee concerning the plausibility of long-term predicted state occupancy, $\log$ (time) was used in the final models. Baseline values of model probabilities used for estimating the clinical consequences of the alternative scenarios were 0.17 for NYHA I, 0.84 for NYHA II and 0.00 for NYHA III (9).

Mortality was low in the REVERSE-EU cohort. Therefore, to maximize the amount of information available to inform parameterization, all follow-up data for European patients initially randomized to the CRT-ON arm were used. For patients in the CRT-OFF arm only data for the first 2 years post-randomization were available to inform model parameters.

A range of parametric survival functions were fitted to timeto-event data with using the treatment as a covariate. On the basis of estimated log-likelihood values, a Weibull proportional hazard model was used.

Utilities. To translate LYG to QALY, a 0.93 (IC; 0.912; 0.96) utility value was assigned for NYHA I patients, 0.78 (IC; $0.722 ; 0.842$ ) for NYHA II patients and 0.61 (IC; $0.591 ; 0.631$ ) for NYHA III patients (12).

Costs. Device costs were taken from Eucomed Q2 2009-Q1 2010 (13). Individuals in the CRT-OFF arm who received a CRT-D device were allocated the cost of an ICD implant. The device lifetime assumed was 6.5 years for CRT, 5.5 years for CRT-D, and 6 years for ICD. The cost of all subsequent device replacements was included in the model.

Because no data have been published on the Spanish costs for the therapeutic options analyzed in this model, a microcosting strategy was used. Data on resources used were obtained from three Spanish cardiologists (Table 1), while unit costs were retrieved from the Spanish Health Costs Database (14) and from the Spanish Drug Price Database (15). Costs were inflated to 2010 values, when necessary.

\section{Sensitivity Analysis}

A probabilistic sensitivity analysis was carried out with all the parameter ranges from the model. A Monte Carlo 


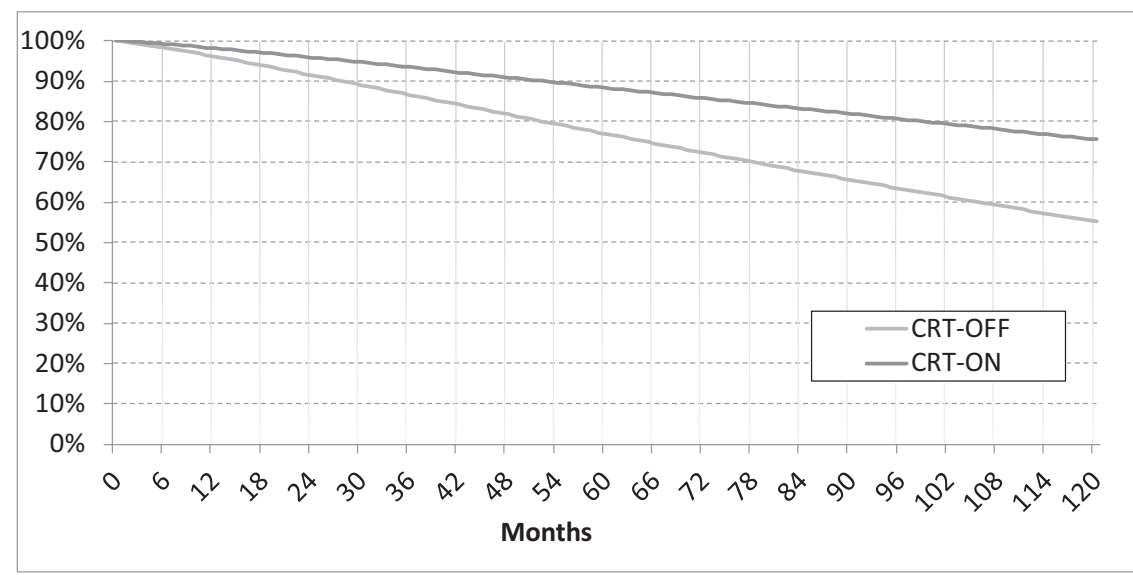

Figure 2. Survival Analysis of CRT-ON and CRT-OFF. CRT, cardiac resynchronization therapy.

simulation analysis was performed, estimating costs and benefits for a cohort of 10000 hypothetical patients to display the cost-effectiveness plane, and building separate acceptability curves to illustrate uncertainty. Current recommendations were followed to derive the parameter distributions: method-ofmoments fitting was used to derive beta distributions for probabilities based on frequency; because costs in the model are mainly a function of the unit cost of the different surgical procedures, lognormal distributions were derived from total intervention costs assuming a standard deviation of 10 percent from mean values obtained using a microcosting strategy.

\section{RESULTS}

\section{Use Resources and Costs}

The introduction of CRT into the management of patients with NYHA functional classes I and II HF provides and additional cost of $€ 16,629.23$ over the 10 -year period post-implantation.

However, the results of the hospitalizations analysis show that the introduction of CRT reduces the length of stay in intensive care unit (ICU) in 94 percent and the stay in general ward in 34 percent. The average days in ICU is 0.091 for CRT-OFF patients and 0.006 for CRT-ON patients, and in the same way, the average length of stay in general ward is 0.705 and 1.076 for CRT-ON and CRT-OFF, respectively.

Overall survival at 10 years is 77.5 percent and 88.2 percent for individuals in the CRT-OFF and CRT-ON arms respectively (Figure 2). CRT-ON patients are in a mild NHYA longer than CRT-OFF patients.

\section{Efficiency and Cost-Effectiveness results}

The Number Needed to Treat to save one life was 4.9, to avoid a day in ICU 1,084 and to avoid a day in general ward 204.

After 6 years, CRT-ON was cost-effective, except for the 7 th year due to device replacement costs for battery depletion. In a 6-year period the results of the cost-effectiveness analysis
Table 2. Incremental Cost-Effectiveness Ratio of Cardiac Rresynchronization Therapy at 10 Years

\begin{tabular}{llcc}
\hline & Life-year gained & QALYS & Costs \\
\hline CRT-OFF & 6.74 & 5.45 & $€ 23,177.30$ \\
CRT-ON & 7.64 & 6.23 & $€ 39,806.53$ \\
Difference & 0.90 & 0.77 & $€ 16,629.22$ \\
Cost per LY gained & & $€ 18,430.92$ & \\
Cost per QALY gained & & $€ 21,500.48$ & \\
& & & \\
\hline
\end{tabular}

CRT, cardiac resynchronization therapy; LY, life-year; QALYS, quality-adjusted life-years.

demonstrate an ICER of $€ 1,142 /$ LYGs and an ICER in terms of QALYs of $€ 11,508 /$ QALYs for patient management with CRT. The cost-effectiveness analysis (Table 2) shows that the introduction of CRT provides an additional effectiveness of 0.90 LYGs per patient in a 10 -year period compared with non-CRT patients. This value results in an ICER of $€ 18,430.92 / L Y G s$. The corresponding gain in terms of QALYs is 0.77 , with an ICER of $€ 21,500.48 /$ QALY.

Supplementary Figure 1, which can be viewed online at www.journals.cambridge.org/thc2013085, shows the acceptability curves of CRT-ON versus CRT-OFF scenarios. Using the defined Spanish criteria of efficiency threshold for a new technology (around $€ 39,000$ per QALY, data from year 2001 adjusted to 2010) (16), the probability of the introduction of CRT being cost-effective is 75.4 percent for CRT-ON patients. Moreover, for thresholds of $€ 30,000$, $€ 35,000$ and $€ 45,000$, the probability of a cost-effective introduction of CRT is 65.54 percent, 71.9 percent, and 79.47 percent, respectively.

Robustness of results was tested varying individual parameter values on model results. The model was sensitive to changes in the proportions receiving an ICD and the associated costs of implantation. Several alternative approaches to modeling 
the REVERSE trial data are explored, with none resulting in an ICER above $€ 34,000$ per QALY gained. The results of the probabilistic analysis show that taking into account the uncertainty in the main variables of the Markov Model, starting the treatment of OMT with CRT remains cost-effective versus the other alternatives.

\section{DISCUSSION}

The use of CRT added to OMT represents an efficient use of resources and a favorable option in patients suffering from heart failure in NYHA functional classes I and II, with costeffectiveness ratios below the Spanish threshold from the 6th year, except for the 7 th year due to device replacement cost for battery depletion.

Both efficacy and effectiveness for CRT had been widely assessed and demonstrated for advanced congestive HF (classes III and IV) unlike for NYHA classes I/II. In general, the efficiency of CRT-P on NYHA III/IV patients is well known, with several studies demonstrating an acceptable ICER against pharmacological treatment such as COMPANION (17), or an extensive cost-effectiveness analysis performed by NICE (National Institute for Clinical Excellence) (18) with 5 years cumulative data from several CRT studies (MIRACLE [19], CONTAK-CD [20], MUSTIC-SR [21], CARE-HF [7;22], and COMPANION [17]). Costs and benefits were derived from a Markov model developed from the British NHS.

Additionally, Callejo et al. (6) evaluated the incremental cost-effectiveness of CRT alone and CRT with implantable defibrillator compared with standard care from the Spanish healthcare perspective. CRT-P appeared to be cost-effective while CRT-D ICER was above the accepted threshold ( $€ 54,000)$.

Previous analyses of the COMPANION (17), CARE-HF $(7 ; 22)$ trials and a Spanish adaptation of CARE-HF trials indicated that both CRT-P and CRT-D devices are cost-effective in patients with NYHA class III/IV (23). Muñoz et al. (23) observed that patients treated with CRT+Standard Care showed an average increase of 0.44 QALYs per patient, resulting in an incremental cost-effectiveness ratio of $€ 14,877$ per QALY gained at 5 years from the Spanish Health System perspective.

The effectiveness data from patients in our study is based on one clinical trial. However, there are other studies, as MADITCRT (10) and RAFT (24), showing a clinical improvement in the introduction of CRT into slighter functional classes as well. MADIT-CRT enrolled 1,820 patients in NYHA function class I (15 percent) of ischemic etiology or II ( 84 percent) of any etiology and sinus rhythm. Using a 2:3 randomization scheme, 731 patients were assigned to receive an ICD and 1,089 received a CRT-D. During a mean follow-up of 2.4 years, the relative risk of sustaining a primary endpoint (death from any cause and non-fatal HF-related adverse events) was reduced by 34 percent in the CRT-D treated group, a benefit attributable primarily to a 41 percent decrease in HF-related adverse events. The 3 percent annual mortality was similar in both REVERSE and MADITCRT studies groups.

At present time, Linde et al. (11) is the only costeffectiveness analysis developed for patients with mild HF from the British NHS perspective. Our study was based on the British model, therefore, the structure and methods are pretty similar to the British study, although in our study the proportion of patients with ICD was greater with CRT-ON (75 percent versus 66 percent) and lower with CRT-OFF (60 percent versus 72 percent). Compared with the Spanish analysis, the results observed in both studies are similar, being the base case ICER in the Linde et al. (11) study $€ 12,172$ per LYG, and $€ 14,278$ per QALY gained. The differences observed could be explained by the fact that the relationship between unitary costs and drug treatments differ among countries. Although we have included general practice visits and more outpatient visit in NYHA III (3 versus 2 visits), Spanish costs per group were lower than British costs, except in CRT group. This difference may be explained by device and leads cost. On the other hand, our analysis included consumption of statins (62 percent to 67 percent), antiplatelet (50 percent to 58 percent), anticoagulant (12 percent to 33 percent), nitrates ( 17 percent to 27 percent), calcium antagonist (10 percent to 12 percent) and amiodarone ( 5 percent to 10 percent) that Linde et al. (11) study did not include.

The ICERs presented in this study are higher than those derived from the CARE-HF study $(7 ; 22)$. This is likely to be a result of a 10-year rather than lifetime time horizon. If the model would have been run for a lifetime the increased benefit associated to delayed progression for NYHA III/IV would have become more apparent. However, further work is required to prove this hypothesis.

It is important to emphasize that the utilities used in our model were different from those used by Callejo et al. (6), and these differences could explain the divergence between conclusions of both studies (25). Utility values used in this analysis are based on observed utilities in United Kingdom (12), although they are considered to be internationally feasible. However, utilities may be considered as a limitation in this study. Keeping in mind the main use of measures related to quality of life in HF that have been used as an outcome in clinical trials, extrapolating the study to the clinical practice could be difficult. Moreover, in most clinical trials a single specific questionnaire was used, usually the Minnesota Living with Heart Failure Questionnaire, which may have hampered a broader vision of impaired Health Related Quality of Life in this syndrome.

Several limitations are present in our study. First, we extrapolate used effectiveness data from the REVERSE-EU study. Therefore, main limitations come from assuming that the clinical practice studied in the REVERSE trial was equal to the Spanish clinical practice, and second, from presuming that this therapy could be incorporated as a treatment for patients with mild to moderate HF in Spain. However, clinical practice in Spain for these patients is similar to that in Europe, 
and patient's age and clinical characteristics are similar, presenting a high level of coincidence with those observed in the REVERSE-EU clinical trial. As a consequence, the results exposed through this study are likely to be close to reality.

Other limitation of the study could possibly lie in the perspective of an expert panel to adapt the use of resources. To avoid potential bias, the use of resources was adapted for Spain. Even more, three Spanish centers participated in the REVERSE trial with an inclusion rate higher than other countries. On the other hand, because the Spanish Cardiology Society does not have specific guidelines for resynchronization, physicians based their daily practice on the ESC guidelines where CRT is recommended for NHYA II. Additionally, CRT is being implanted within this subgroup of patients with an implantation rate considerably lower than other U.S. or EU countries, mainly due to CRT initial costs, which represented a strong barrier for the adoption of this therapy.

Our study only took into account direct costs incurred by the Spanish healthcare system, but in real practice other costs associated with or derived from the society do exist, like temporary sick leaves or early retirements (productivity loss). Therefore, should this study have considered indirect costs or the slowing down of the disease progression resulting from an earlier incorporation of CRT, the ICERs would have been lower, and thus CRT would have been considered a more efficient alternative.

A Spanish efficiency threshold for a new technology is not defined, although the values assumed correspond to $€ 30,000 / \mathrm{QALY}$ and $€ 30,000 / \mathrm{LYGs}$ (€ 2001) (16), proving that CRT is a cost-effective intervention. Cost-effectiveness studies are valuable tools for comparing different therapeutic alternatives and, therefore, are useful for decision making in the framework of overall assessment of medical interventions and healthcare technologies.

\section{CONCLUSION}

Finally, our results demonstrate that the implantation of CRT$\mathrm{D}$ in earlier stages of the disease represents a cost-effective treatment alternative in patients with mildly symptomatic left ventricular dysfunction and $\mathrm{QRS} \geq 120 \mathrm{~ms}$ from the perspective of the Spanish Health System.

\section{SUPPLEMENTARY MATERIAL}

Supplementary Figure 1:

www.journals.cambridge.org/

thc 2013085

\section{CONTACT INFORMATION}

Luis Almenar, Cardiology Department, Hospital Universitario La Fe, Valencia, Spain

Beatriz Díaz, Cardiology Department, Hospital Universitario Central de Asturias, Oviedo, Spain
Aurelio Quesada, Cardiology Department, Hospital General de Valencia, Spain

Carlos Crespo, Department of statistics, University of Barcelona, Oblikue Consulting, Barcelona, Spain

Belén Martí, (belen.marti@medtronic.com), Health Economics and Reimbursement, Medtronic Ibérica, Madrid, Spain Stuart Mealing, Oxford Outcomes, United Kingdom

Cecilia Linde, Department of Cardiology, Karolinska University Hospital, Stockholm, Sweden

Claude Daubert, Département de Cardiologie et maladies vasculaires, CHU, Rennes, France

\section{CONFLICTS OF INTEREST}

Carlos Crespo reports funding, including payment for manuscript preparation, to himself and his institution from Oblikue consulting. Belen Marti is a full-time employee of Medtronic. Stuart Mealing reports funding to his institution from Oxford Outcomes UK. Cecilia Linde reports a grant to her institution from Medtronic and consulting fees to herself from Cardiomems, MSD and Swedish Heart Lung foundation.

\section{REFERENCES}

1. Dickstein K, Cohen-Solal A, Filippatos G, et al. European Society of Cardiology (ESC) Guidelines for the diagnosis and treatment of acute and chronic heart failure 2008. Eur Heart J. 2008;29:2388-2442.

2. Banegas JR, Rodríguez-Artalejo F, Guallar-Castillón P. [Epidemiological situation of heart failure in Spain]. Rev Esp Cardiol. 2006;6(Suppl C):4-9.

3. National Statistics Institute (INE). Deaths by cause of death 2008. www.ine.es (accessed June 1st, 2010).

4. Sánchez V, Cavero MA, Delgado JF, Pulpón LA. [Cardiac resynchronization therapy: Point of view of clinical cardiologist]. Rev Esp Cardiol. 2005;5(Suppl B):53-59.

5. Gomez-Soto FM, Andrey JL, García-Egido AA, et al. Incidence and mortality of heart failure: A community-based study. Int $J$ Cardiol. 2011;151:40-45.

6. Callejo D, Guerra M, Hernández-Madrid A, Blasco JA. Economic assessment of cardiac resynchronization therapy. Rev Esp Cardiol. 2010;63:1235-1243.

7. Calvert MJ, Freemantle N, Yao G, et al. Cost-effectiveness of cardiac resynchronization therapy: results from the CARE-HF trial. Eur Heart J. 2005;26:2681-2688.

8. Silva L, García-Pavía P, Ortigosa J. [Resynchronization and prevention of sudden death in heart failure. From clinical trials to clinical practice]. Rev Esp Cardiol. 2006;6(Suppl F):59-70.

9. Daubert C, Gold MR, Abraham WT, et al. Prevention of disease progression by cardiac resynchronization therapy in patients with asymptomatic or mildly symptomatic left ventricular dysfunction: insights from de European cohort of REVERSE (Resynchronization Reverses Remodeling in Systolic Left Ventricular Dysfunction) trial. J Am Coll Cardiol. 2009;54;1837-1846.

10. Moss AJ, Jackson W, Cannom DS, Klein H, Brown MW, MADIT-CRT trial investigators. Cardiac-resynchronization therapy for the prevention of heart failure events. N Engl J Med. 2009;361:1329-1338.

11. Linde C, Mealing S, Hawkins N, et al. Cost-effectiveness of cardiac resynchronization therapy in patients with asymptomatic to mild heart failure: insights from the European cohort of the REVERSE (Resynchronization Reverses remodeling in Systolic Left Ventricular Dysfunction). Eur Heart J. 2011;32:1631-1639. 
12. Fox M, Mealing S, Anderson R, et al. The clinical effectiveness and costeffectiveness of cardiac resynchronisation (biventricular pacing) for heart failure: systematic review and economic model. Health Technol Assess. 2007;11:11-68, iii-iv, ix-248

13. EUCOMED. Medical device price report Q2 2009-Q1 2010. www. eucomed.org (accessed June 1st, 2010).

14. E-salud. Spanish health cost data base. Barcelona, 2010. http://www. oblikue.com (accessed June 1st, 2010).

15. Spanish General Council of Official Colleges of Pharmacists. Spanish drug price database. Bot Plus Web, 2010. https://botplusweb.portalfarma. com/ (accessed June 1st, 2010).

16. Sacristán JA, Oliva J, Del Llano J, Prieto L, Pinto JL. What is an efficient health technology in Spain? Gac Sanit. 2002;16:334343.

17. Feldman AM, de Lissovoy G, Bristow MR, et al. Cost effectiveness of cardiac resynchronisation therapy in the Comparison of Medical Therapy, Pacing, and Defibrillation in Heart Failure (COMPANION) trial. $J$ Am Coll Cardiol. 2005;46:2311-2321.

18. National Institute for Clinical Excellence (NICE). Cardiac resynchronization therapy for the treatment of heart failure. NICE technology appraisal guidance 120. 2007. www.nice.org.uk/TA120 (accessed June 1st, 2010).

19. St John Sutton MG, Plappert T, Abraham WT, et al. Effect of cardiac resynchronization therapy on left ventricular size and function in chronic heart failure. Circulation. 2003;107:1985-1990.

20. Higgins SL, Hummel JD, Niazi IK, et al. Cardiac resynchronization therapy for the treatment of heart failure in patients with intraventricular conduction delay and malignant ventricular tachyarrhythmias. $\mathrm{J} \mathrm{Am} \mathrm{Coll}$ Cardiol. 2003;42:1454-1459.

21. Alonso C, Ritter P, Leclercq C, et al. Effects of cardiac resynchronization therapy on heart rate variability in patients with chronic systolic heart failure and intraventricular conduction delay. Am J Cardiol. 2003;91:11441147.

22. Yao G, Freemantle N, Calvert MJ, et al. The long term cost-effectiveness of cardiac resynchronization therapy with or without an implantable cardioverter-defibrillator. Eur Heart J. 2007;28:42-51.

23. Muñoz R, Martínez-Ferrer J, Delgado J, et al. [Can cardiac resynchronization therapy added to optimize pharmacological treatment make up in patients with heart failure in Spain?]. PharmacoEconomics Sp Res Art. 2010;7:13-25.

24. Tang AS, Wells GA, Talajic M, et al. Resynchronization-defibrillation for ambulatory heart failure trial investigators. Cardiac-resynchronization therapy for mild-to-moderate heart failure. N Engl J Med. 2010;363:23852395.

25. Calvert MJ, Freemantle N, Cleland JG. Cardiac resynchronisation therapy is cost effective. Rev Esp Cardiol. 2010;63:1230-1231. 